\title{
High-Gyrotropy Seedlayer-Free Ce:TbIG for Monolithic Laser- Matched SOI Optical Isolators
}

\author{
Karthik Srinivasan, ${ }^{* \dagger \odot}$ Cui Zhang, ${ }^{\ddagger}, \bigcirc$ Prabesh Dulal, ${ }^{\S} \square$ Cosmin Radu,, Thomas E. Gage, ${ }^{\S} \triangle$ \\ David C. Hutchings, ${ }^{\neq}$and Bethanie J. H. Stadler* ${ }^{* \dagger, \S}$ \\ ${ }^{\dagger}$ Electrical and Computer Engineering, University of Minnesota, Minneapolis, Minnesota 55455, United States \\ ${ }^{\ddagger}$ James Watt School of Engineering, University of Glasgow, Glasgow G12 8LS, United Kingdom \\ ${ }^{\S}$ Chemical Engineering and Materials Science, University of Minnesota, Minneapolis, Minnesota 55455, United States \\ "Lakeshore Cryotronics Inc., Westerville, Ohio 43082, United States
}

\section{Supporting Information}

ABSTRACT: Monolithic optical isolators that provide modal (transverse electric, TE) and dimensional (500 $\mathrm{nm}$ core) matching to on-chip lasers have been realized with "one step" seedlayer-free garnets. To date, seedlayer-free garnet claddings have required thinner $(<340 \mathrm{~nm})$ silicon-on-insulator (SOI) cores because mode-cladding interactions were too weak for laser-matched cores. However, laser matching is important because tapers and mode converters between the laser and the isolator can cause detrimental reflections prior to isolation. This paper reports the use of cerium-doped terbium iron garnet (Ce:TbIG) in a quasi-phase matched nonreciprocal mode conversion (NRMC) isolator that operates on both TE

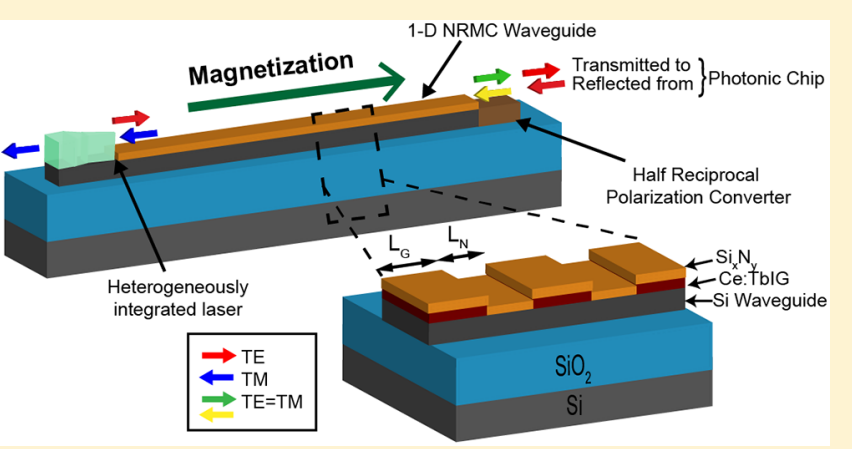
and TM modes without an external field. A key innovation presented here is a repeatable process for foundry-friendly sputter deposition of Ce:TbIG, which enables this high Faraday rotation material $(-3200 \% \mathrm{~cm})$ to be synthesized in any isolator design that would benefit from one-step lithographical manufacturing. A proof-of-feasibility $500 \mathrm{~nm}$ SOI NRMC device is demonstrated with seedlayer-free Ce:TbIG that achieves an isolation ratio of $11 \mathrm{~dB}$. With an optimal length, this NRMC design can provide greater than $30 \mathrm{~dB}$ isolation.

KEYWORDS: magneto-optical garnets, isolators, silicon photonics, Faraday rotation, non-reciprocal mode conversion, cerium-doped terbium iron garnet, magnet-free isolators

Cilicon-on-insulator (SOI) photonic integrated circuits $\checkmark$ (PICs) are now manufactured with several hundred devices each, similar to early electronic ICs. ${ }^{1,2}$ However, these SOI PICs still suffer from a lack of deployed solutions for incorporating isolators (or optical "diodes") to protect integrated laser sources. ${ }^{3}$ Current prototype integrated isolators have dimensional mismatch ${ }^{4,5}$ and mode incompatibility with on-chip laser sources. ${ }^{6-14}$ Dimensional mismatch can, in principle, be accommodated with tapers and, mode (or polarization) incompatibility can be overcome by complex infrastructures, such as polarization converters and couplers, but generally each component and interface can cause unwanted reflections before isolation.

Dimensional mismatch occurs because integrated hybrid lasers require $>400 \mathrm{~nm} \mathrm{Si}$ cores to couple light into SOI waveguides, ${ }^{4}$ but current $\mathrm{Si}$ waveguide isolators have mostly exploited the transverse magneto-optic (MO) effect, which requires $220 \mathrm{~nm}$ SOI with magneto-optic garnet top claddings. $4,6,8,15-17$ Specifically, a nonreciprocal phase shift (NRPS) occurs for the transverse magnetic (TM) modes, but these designs are fundamentally limited by the need for asymmetric interaction of evanescent waves in the garnet (upper) and silica (lower) claddings. This is also applicable for TE-mode devices with sidewall coatings that require narrow widths for the same reason TM NRPS devices need thin cores and, as a result, will also normally require thin cores. Consequently, extremely high confinement is essential, and NRPS falls off rapidly if the core thickness increases beyond $220 \mathrm{~nm}$. This thickness matches most components in the PIC chip because thin waveguides can be easily designed for fundamental mode operation. ${ }^{18-21}$ Unfortunately, neither TMmode operation nor $220 \mathrm{~nm}$ cores match integrated lasers, which are TE-mode and have a typically $500 \mathrm{~nm}$ thick core. Therefore, mode converters and tapers will be required before the isolator, and detrimental reflections occur before they can be isolated from impinging back on the laser, see "standard isolators" in Figure $1 .^{22}$

An alternative to NRPS that enables $500 \mathrm{~nm}$ SOI core isolators is a nonreciprocal mode conversion $(\mathrm{NRMC})^{9}$ via a

Received: May 15, 2019

Published: September 6, 2019 


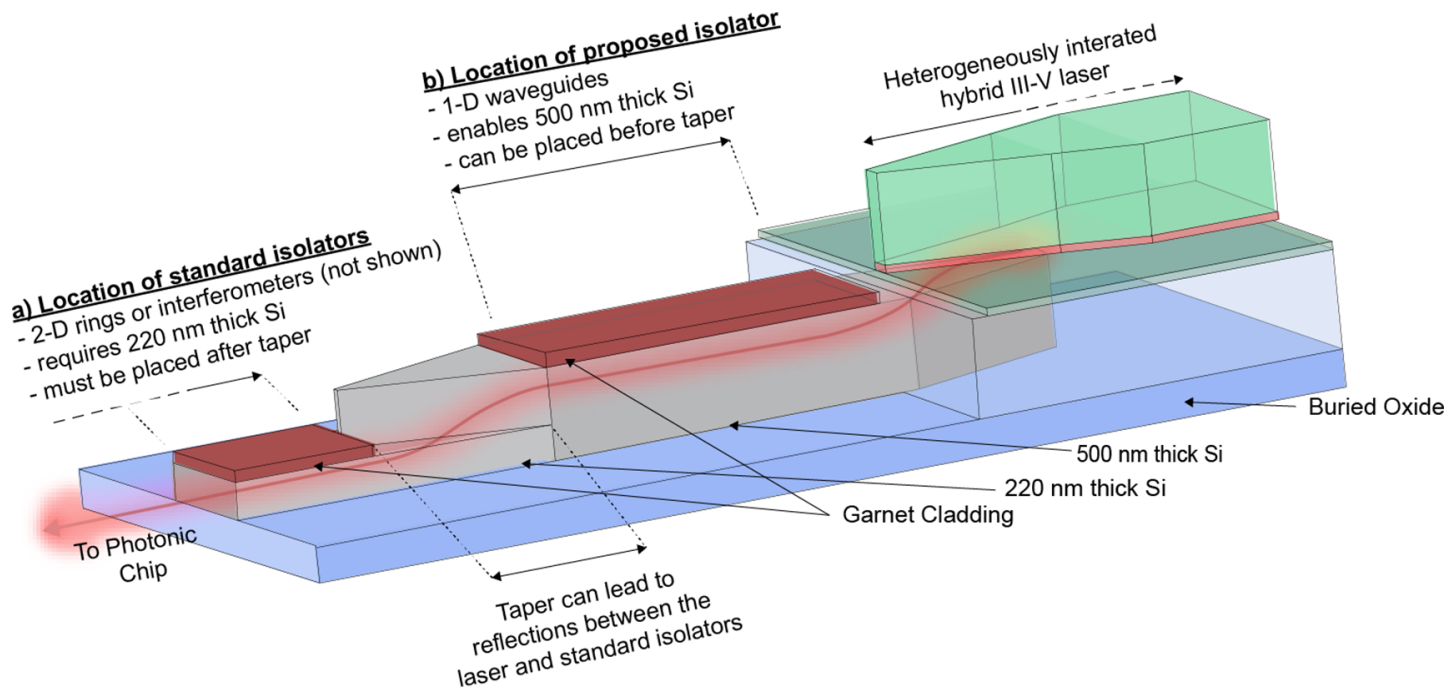

Figure 1. Schematic showing isolators in current literature, placed after tapering from the laser region to 220 nm SOI for NRPS functionality, compared to the isolators described in this paper, placed immediately after the laser coupling region. The location of the proposed 1D NRMC isolator ensures isolation of all the reflections from tapers or device interfaces, such as polarization converters used in both NRMC and NRPS devices. See refs 4 and 21 as examples of III-V laser integration, which is not shown in detail here.

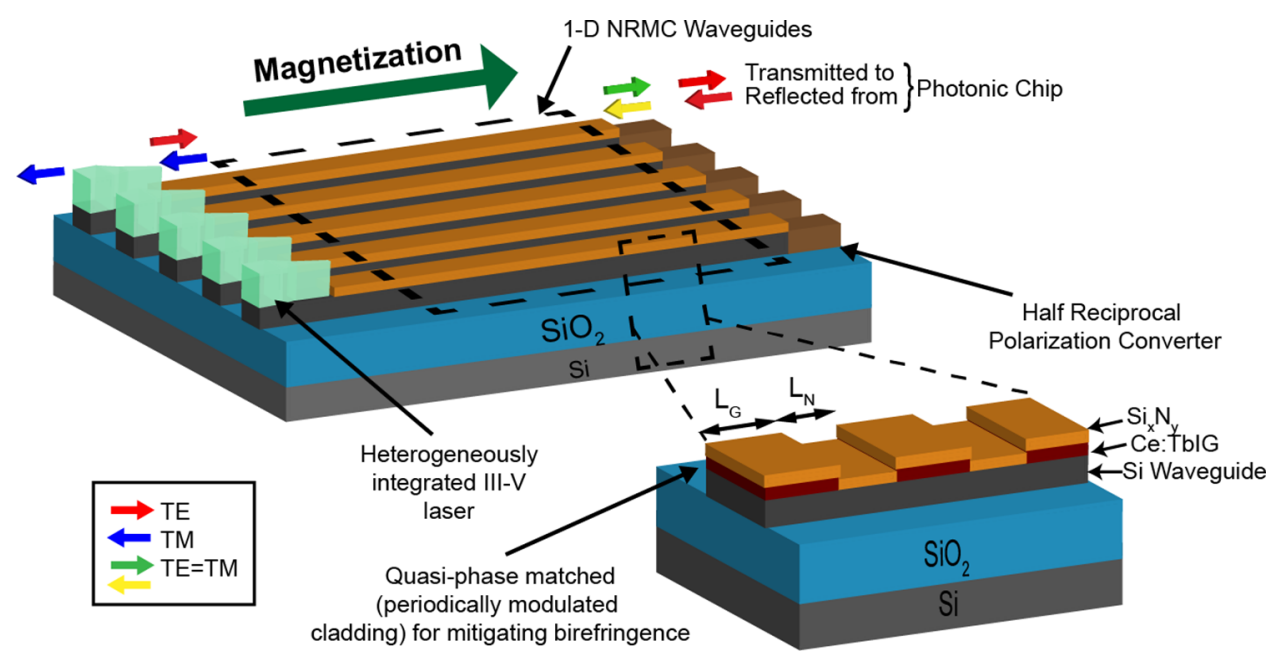

Figure 2. Schematic of $1 \mathrm{D}$ waveguide isolator details with segmented cladding of Ce:TbIG $/ \mathrm{Si}_{X} \mathrm{~N}_{Y}$ to obtain nonreciprocal mode conversion (NRMC), which is the waveguide equivalent of Faraday rotation. A half reciprocal phase converter converts the TE $=\mathrm{TM}$ mode output into TE modes for forward propagation into the photonic circuit and vice versa for backward propagation. Colored arrows are used to show polarization as shown in the Stokes sphere, Supporting Information (SI; Figure S1).

garnet top cladding. NRMC is the waveguide equivalent of Faraday rotation, which is used in conventional benchtop and fiber-coupled isolators. ${ }^{23,24}$ The evanescent NRMC interaction with the garnet cladding reduces as the core dimension increases, but it falls off slowly compared to the critical asymmetry criterion in NRPS devices. Therefore, if a garnet with sufficient gyrotropy is found and coupled with proper SOI isolator design, an integrated isolator may finally be feasible where isolation occurs before any tapers or additional interfaces, see "proposed isolator" in Figure 1. This paper reports on the first combination of one-step lithography garnets with a photonic design that will enable protection for integrated lasers without an external magnetic field.

In addition, the presented design mitigates the mode incompatibility of current isolators because NRMC operates on TM and TE modes, and isolation is achieved with the simple addition of a half reciprocal polarization converter $(\mathrm{H}-$ RPC) and a longitudinal magnetic field, Figure 2. In contrast,
NRPS has only achieved TE isolation by using full polarization converters at both the input and output of the isolator ${ }^{7}$ and, recently, by sidewall coating. ${ }^{25}$ Another advantage of NRMC devices is that they are one-dimensional (1D), with very small footprints, Figure 2, unlike NRPS devices that are twodimensional (2D), requiring an interferometer or ring resonator to utilize the phase shift.

Some researchers have thought it would be difficult to utilize NRMC in SOI platforms due to the birefringence of silicon waveguide structures. ${ }^{26,27}$ However, quasi-phase matching (QPM) has been shown to overcome birefringence. ${ }^{28,29}$ QPM is achieved using alternating MO and non-MO segments with lengths that match the characteristic beat length of the waveguide birefringence, inset Figure 2. Therefore, not only can NRMC designs use $500 \mathrm{~nm}$ thick SOI, they actually benefit from thicker waveguides because cross sections will be more isotropic. Also, QPM structures can be fabricated via 

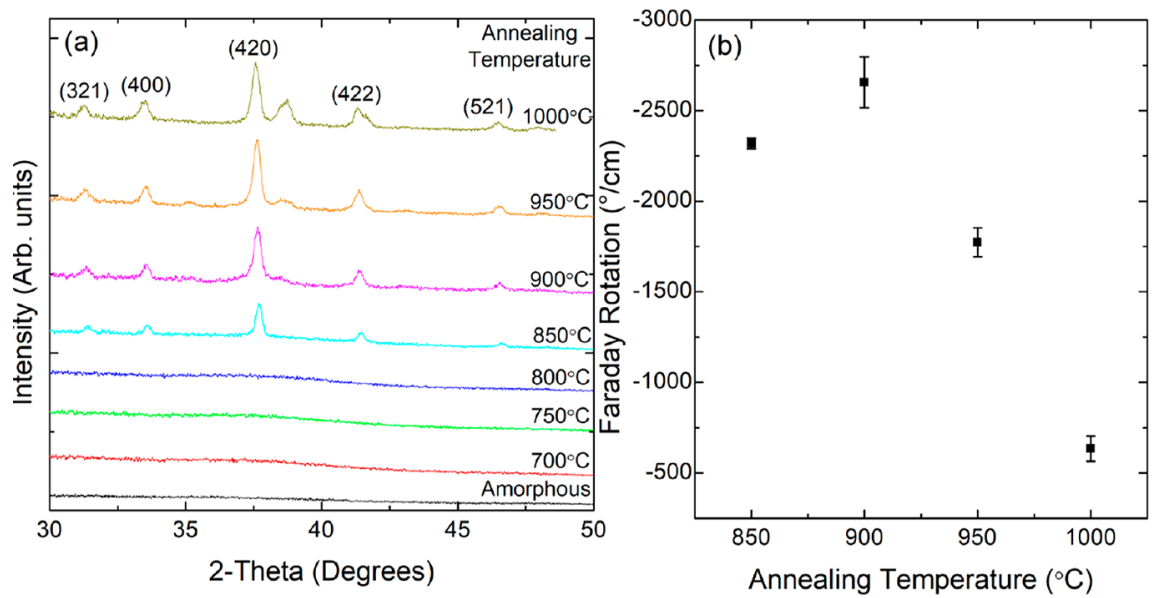

Figure 3. Optimization of annealing temperature. (a) X-ray diffraction spectra obtained for Ce:TbIG samples grown at $40 \mathrm{~W}$ cerium sputtering power and annealed at temperatures from 700 to $1000{ }^{\circ} \mathrm{C}$ for $3 \mathrm{~min}$. Optimized crystallization of the garnet phase occurs at $900{ }^{\circ} \mathrm{C}$, after which secondary phases appear (garnet peaks are identified in parentheses). (b) A correspondingly high Faraday rotation was obtained for the sample annealed at $900{ }^{\circ} \mathrm{C}$ (the error bars are calculated from the variations obtained in three consecutive measurements).
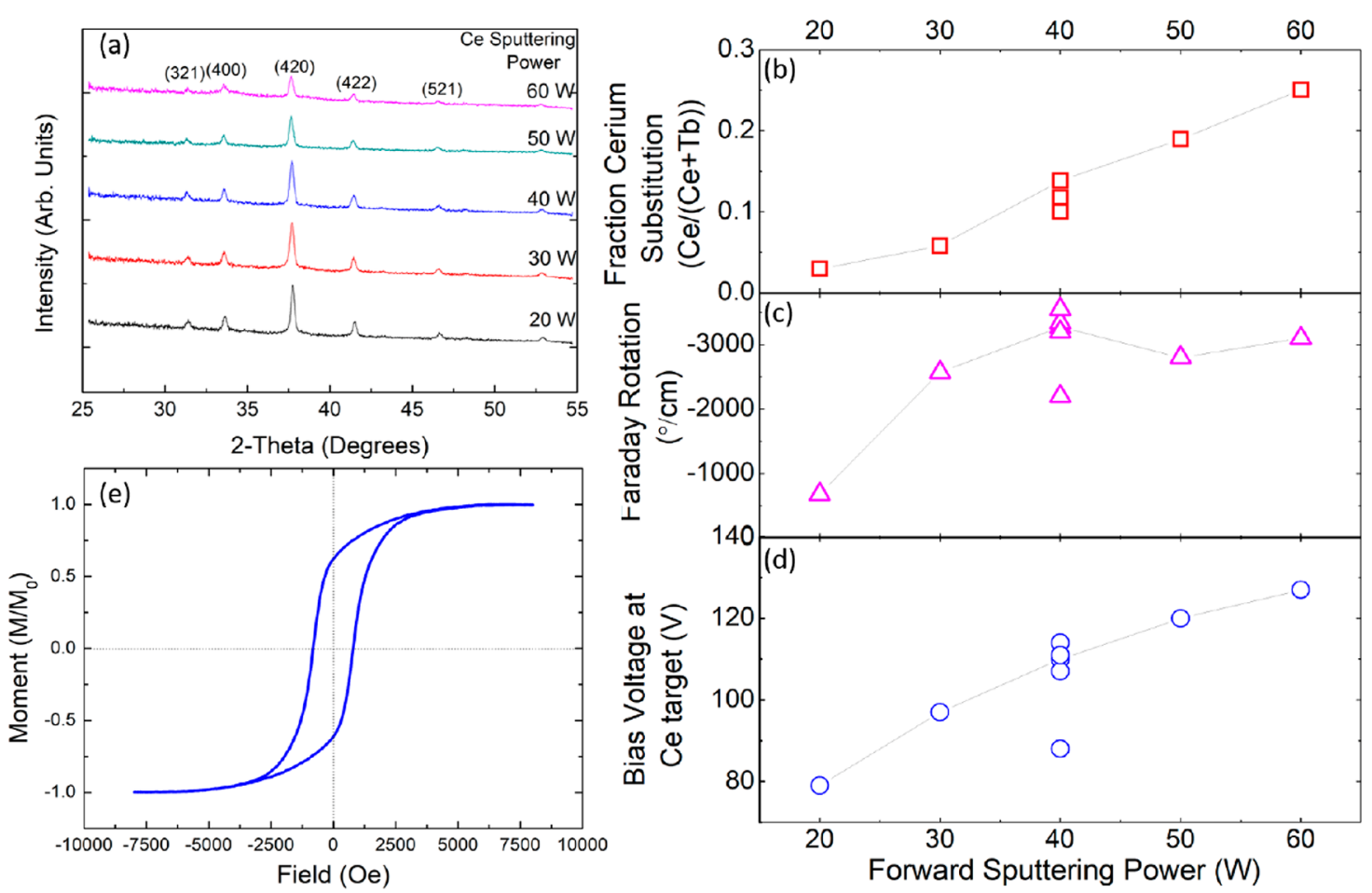

Figure 4. Optimization of Ce-doping. (a) XRD spectra of Ce:TbIG annealed at $900{ }^{\circ} \mathrm{C}$ with various cerium dopant concentrations corresponding to change in Ce sputtering power from 20 to $60 \mathrm{~W}$ (garnet peaks are identified in parentheses). (b) Increase in fraction cerium substitution (Ce/ $(\mathrm{Ce}+\mathrm{Tb}))$ with higher cerium sputtering powers. (c) Faraday rotation measurements show that very large rotation values $\left(>-3200^{\circ} / \mathrm{cm}\right)$ are obtained at $40 \mathrm{~W}$ of forward sputtering power. The outlier is the exception with low Faraday rotation. (d) Variation of the bias voltage at cerium target for different sputtering powers. The distribution of bias voltages at $40 \mathrm{~W}$ is comparable to the distribution of Faraday rotation at $40 \mathrm{~W}$. The outlier corresponds to the low rotation sample. (e) Room temperature M-H loop for the in-plane measurement directions shows desirable magnetic properties for an optimal Ce:TbIG sample sputtered at $40 \mathrm{~W}$ Ce.

photolithography or nanoimprint rather than expensive e-beam lithography for foundry-friendly designs.

The question remaining is how to incorporate a high gyrotropy garnet cladding onto the SOI waveguides in order to produce an isolator. Fortunately, Ce:TbIG has been found to crystallize on top of $\mathrm{Si}$ without an optically detrimental seedlayer that is needed for other garnets (e.g.: doped YIG). ${ }^{30-34}$ Unfortunately, high gyrotropy has been difficult to obtain repeatedly in sputter-deposited Ce:TbIG compared to other sputter-deposited garnets. Here, we report a repeatable, foundry-friendly $\mathrm{Ce}$ :TbIG process accomplished by optimizing annealing temperature and Ce-doping. ${ }^{30,35}$ Importantly, the NRMC materials presented here could also be useful for any passive integrated isolator, including NRPS devices.

It is important to mention that future integrated photonic circuits may migrate to thicker cores for several reasons. First, as mentioned here, the integrated lasers available require 500 
(a)

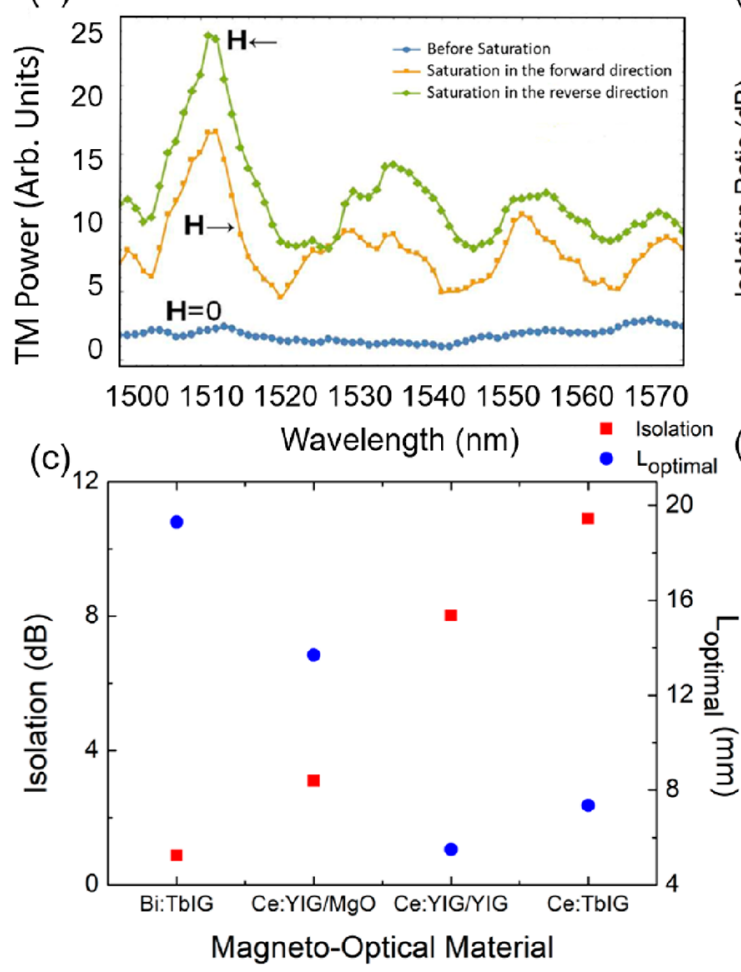

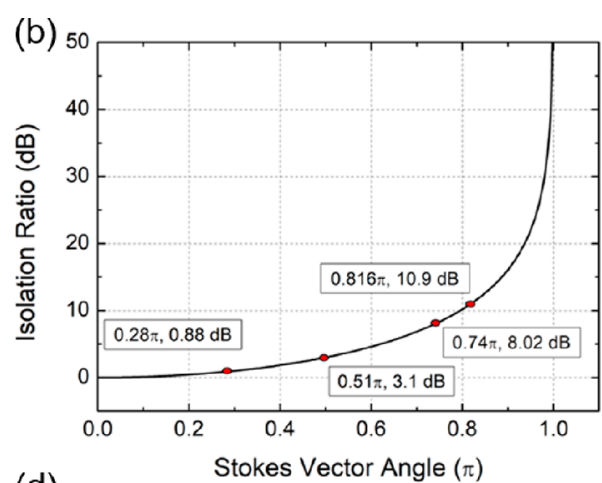

(d)

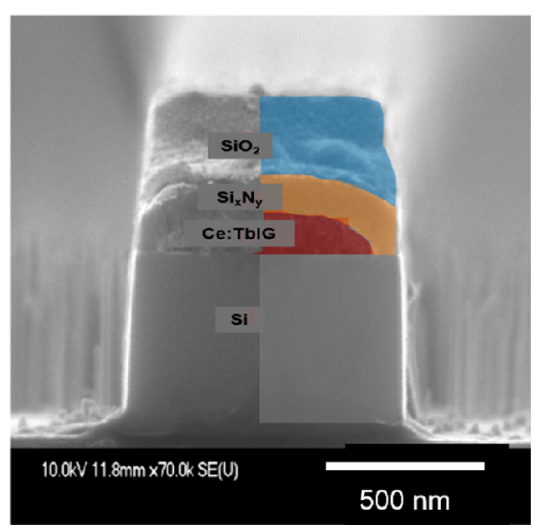

Figure 5. Nonreciprocal mode conversion measurements. (a) Wavelength scan to detect the TM mode output with TE mode laser source. The peak in output percentage for $1511 \mathrm{~nm}$ corresponds to TE to TM mode conversion due to the Faraday effect. (b) Isolation ratio calculated from the Stokes vector angle shows that for $\pi$ radians the isolation ratio is maximum. The values measured for devices in Table 1 are superimposed on the theoretical result. (c) Comparison of isolation and optimal length of the device for different magneto-optical cladding materials. The device with Ce:TbIG cladding has the largest isolation ratio. (d) Cross-section scanning electron microscope (SEM) image of the 500 nm waveguide isolator with Ce:TbIG cladding.

nm SOI. Second, amplifiers, phase modulators, and photodetectors all currently require thick cores. ${ }^{4}$ However, the development of a dual-level SOI platform is extremely challenging, especially the preservation of pristine silicon surfaces for bonding or deposition needed to facilitate low-loss waveguide propagation. It is difficult to meet uniformity and surface smoothness requirements upon partial etching of a thicker SOI core. Alternatively, the selective area deposition of amorphous silicon, as demonstrated by Ghosh et al., ${ }^{18}$ can increase the core thickness from the initial $220 \mathrm{~nm}$ SOI, but could prove problematic when subsequently addressing III-V bonding for the active elements with a thickened core. In these cases, the only viable isolators proposed to date are the NRMC isolators presented here.

\section{RESULTS AND DISCUSSION}

Foundry-Friendly Processing of Seedlayer-Free Ce:TbIG on Si. Until now, Ce:TbIG has proven more difficult to synthesize on Si substrates and waveguides with repeatedly high gyrotropy $\left(>-3200^{\circ} / \mathrm{cm}\right)$. Here, a foundry-friendly process is defined by careful parameter optimization in order to enable high process yields for any integrated isolator that would benefit from one-step lithography, including the demonstration device presented below.

Magnetic gyrotropy in sputter-deposited garnets can be optimized via two routes: crystallization of the garnet phase and control of Ce-doping. A minimum temperature of $850{ }^{\circ} \mathrm{C}$ is required to crystallize the garnets in the useful polycrystalline phase, Figure 3a. Higher annealing temperatures can yield a secondary phase, possibly a hexaferrite phase, which can lead to optical loss and lower Faraday rotation, Figure $3 \mathrm{~b}$. To mitigate thermal damage to other devices, laser annealing can be used to restrict the heated area to the desired cladding position. $^{35}$

It is standard to optimize doping by varying the forward power to the appropriate cathode (in this case, Ce) during reactive cosputter deposition processes, Figure $4 \mathrm{~b}$. Details are discussed in Figure S2. Typically, one increases the favorable dopant to an optimal level that still results in crystallization of the desired phase (in this case, garnet). Figure $4 \mathrm{a}$ and $\mathrm{b}$ show that the garnet phase crystallized with Ce substituting for up to $25 \%$ of the $\mathrm{Tb}$ sites (up to $60 \mathrm{~W}$ forward power). A maximum Faraday rotation (FR) of $-3200 \pm 200 \% \mathrm{~cm}$ was achieved at $40 \mathrm{~W}$ before saturation at higher $\mathrm{Ce}$ content, Figure $4 \mathrm{c}$, probably due to secondary phases that are not visible in the diffraction spectra. The origin of very large FR in Ce:TbIG is attributed to a combination of steric effects and electronic transitions that can enhance Faraday rotation at a particular wavelength and change the ellipticity independent of the contrasting effects of $\mathrm{Tb}$ and Ce ions. ${ }^{36}$ Interestingly, control of the forward power was not enough to ensure repeatability, Figure 4c. In fact, the bias voltage at the Ce target was determined to be the most important factor in obtaining high Faraday rotations, Figure $4 \mathrm{~d}$, where films made at low bias voltages were found to have low gyrotropies. Conveniently, the bias voltage can be monitored and held constant by varying the forward power to produce repeatedly high gyrotropy in Ce:TbIG films. 
Figure $4 \mathrm{e}$ shows the room-temperature in-plane $\mathrm{M}-\mathrm{H}$ loop measurement for Ce:TbIG. With a saturation magnetization$\left(\mathrm{M}_{\mathrm{S}}\right)$ of $18 \mathrm{emu} / \mathrm{cc}$, a large coercivity and remanence along the shape anisotropy direction, this material appears to be an ideal candidate of latched or magnetless isolators. ${ }^{13,23}$ The low $M_{\mathrm{S}}$ is an intrinsic material property at room temperature and is not impacted due to the proximity to the ferrimagnetic compensation temperature ( $248 \mathrm{~K}$ for bulk $\mathrm{TbIG}$ ) of the garnet. ${ }^{37}$ A low saturation magnetization material will also have lower stray fields in contrast to other garnets used in isolator designs whose $M_{\mathrm{S}}$ exceeds $100 \mathrm{emu} / \mathrm{cc}$.

Prototype Waveguide Isolator Devices. The motivation of this work is an integrated isolator design with a $500 \mathrm{~nm}$ SOI core via one-step garnet precursor deposition, meaning no seedlayers. It has been shown that seedlayers are detrimental to mode-cladding interaction, as are bonding layers. ${ }^{29}$ Although successful isolators have used, for example, Ce:YIG on YIG seedlayers, the processing involves multiple lithography and annealing steps. This requires multiple vacuum breaks, careful realignment of photoresist before the Ce:YIG layer is deposited, and an overall high thermal budget.

Here, one-dimensional (1D) waveguide isolators were fabricated with alternating segments of Ce:TbIG cladding on $500 \mathrm{~nm} \times 900 \mathrm{~nm} \mathrm{Si}$ waveguides. Details about the measurements are given in the Supporting Information. The phase-matching beat length was $21 \mu \mathrm{m}(10.5 \mu \mathrm{m}$ Ce:TbIG/ $10.5 \mu \mathrm{m} \mathrm{Si}{ }_{X} \mathrm{~N}_{Y}$ ). Although these structures were defined by ebeam lithography, the dimensions are commensurate with fabrication via photolithography and localized laser annealing for eventual foundry-friendly devices. ${ }^{35}$ Optical losses were estimated at $1550 \mathrm{~nm}$ using the Fabry-Perot technique, giving $\mathrm{TM}$ and TE losses of 14.7 and $11.2 \mathrm{~dB} / \mathrm{cm}$, respectively. Here, the one-way optical loss of $4-6 \mathrm{~dB}$ is only due to the NRMC components, unlike NRPS designs where couplers and polarization converters further add to their insertion losses. An accurate measurement of the material absorption coefficient of a nearly transparent (at $1550 \mathrm{~nm}$ ) garnet is limited by reflections and scattering from substrate or sensitivity of the instrument which is $0.5-1 \mathrm{~dB} / \mathrm{mm}$.

The TE-TM mode conversion efficiency was measured after saturation with and removal of a longitudinal magnetic field of $\sim 1.2 \mathrm{kOe}$. The NRMC peak exhibited the shape of the peak commensurate with the expected $\operatorname{sinc}^{2}$ for phase-matched coherent mode conversions, Figure 5a. The traces before and after saturation do not indicate any TE-TM mode conversion due to periodic loading, which is typically observed before saturation. The mode conversion peak obtained on saturation is fully attributed to the magneto-optical NRMC that allows for effective isolation.

Several magneto-optical garnets were compared by depositing QPM claddings onto $500 \mathrm{~nm}$ SOI waveguides (Table 1). The angles between input and output Stokes vectors $(\Theta)$ and the isolation ratios (IR) were described previously ${ }^{9}$ and in the SI. This difference was measured using cleaved devices, and the optimal length needed for an ideal Stokes vector angle of $\pi$ was calculated. For devices of length $\left(L_{\text {optimal }}\right)$, light waves of opposite propagation direction will be orthogonal to each other at the laser, and $100 \%$ isolation will be achieved. Figure $5 b$ shows that the isolation ratios greater than $30 \mathrm{~dB}$ can be obtained for near ideal Stokes vector angle of $\pi$ radians. The bandwidth for an ideal waveguide isolator is determined by phase-matching of the NRMC interaction. The period of the cladding structure $L_{\mathrm{G}}+L_{\mathrm{D}}$ sets the peak wavelength, and the
Table 1. Summary of the Measured and Calculated Results from Waveguide Isolators with Different Garnet Cladding Fabricated on $500 \mathrm{~nm} \mathrm{SOI}^{a}$

$\begin{array}{lcccc}\text { garnet cladding } & L_{\mathrm{m}}(\mathrm{mm}) & \Theta(\mathrm{rad}) & d_{\mathrm{m}}(\mathrm{dB}) & L_{\text {optimal }}(\mathrm{mm}) \\ \mathrm{Bi}: \text { TbIG } & 5.4 & 0.28 \pi & 0.88 & 19.3 \\ \mathrm{Ce}: \mathrm{YIG} / \mathrm{MgO} & 7.0 & 0.51 \pi & 3.1 & 13.7 \\ \mathrm{Ce}: \text { YIG/YIG } & 4.1 & 0.74 \pi & 8.0 & 5.50 \\ \mathrm{Ce}: \text { TbIG } & 6.0 & 0.82 \pi & 11 & 7.35\end{array}$

${ }^{a}$ In the table, $L_{\mathrm{m}}$, measured device length; $\Theta$, measured Stokes vector angle; $d_{\mathrm{m}}$, calculated isolation ratio; $L_{\text {optimal }}$, projected optimal length needed to achieve a $\Theta$ of $\pi$ radians.

bandwidth is provided by the usual $\operatorname{sinc}^{2}$ phase-matching dependence such that the main lobe is within $\left|\Delta k_{\mathrm{G}} \frac{L_{\mathrm{G}}}{L_{\mathrm{G}}+L_{\mathrm{D}}}+\Delta k_{\mathrm{D}} \frac{L_{\mathrm{D}}}{L_{\mathrm{G}}+L_{\mathrm{D}}}-\frac{2 \pi}{L_{\mathrm{G}}+L_{\mathrm{D}}}\right| L_{\mathrm{dev}}<\pi$. The wavelength tuning modal wavenumber difference $\Delta k$ in each section will be dependent on the waveguide and material dispersions. At present, the unoptimized bandwidth is on the order of a nanometer, however, as in other QPM approaches, the bandwidth can be increased by design with use of aperiodic or chirped gratings. ${ }^{38}$

The most suitable magneto-optical garnet for $500 \mathrm{~nm}$ SOI isolator can be identified by comparing the isolation ratios and the projected $L_{\text {optimal }}$ for the different material choices shown in Figure 5c. Bi:TbIG has been measured to have lower Faraday rotation $\left(-500^{\circ} / \mathrm{cm}\right){ }^{29}$ and yet, QPM claddings could produce full isolation if the chip had room for an isolator that was $2 \mathrm{~cm}$ long. Note, such lengths have been achieved using windings that would equal the size of the $2 \mathrm{D}$ isolator designs found in the literature. ${ }^{7} \mathrm{Ce}: \mathrm{YIG} / \mathrm{MgO}$ claddings produced marginally higher isolation ratios but still need $13.7 \mathrm{~mm}$ to achieve $\pi$ between the Stokes vectors.

Ce:YIG has been measured to have the largest Faraday rotation possible on $\mathrm{Si}$ substrates $\left(-3700^{\circ} / \mathrm{cm}\right),{ }^{33}$ but it requires YIG seedlayers. As expected, QPM claddings of this garnet had considerably better isolation of $8.0 \mathrm{~dB}$ with the shortest optimal length of $5.5 \mathrm{~mm}$. However, the undoped YIG seedlayer required for this garnet also requires a two-step deposition. For the best crystallization of Ce:YIG, two lift-off/ anneal steps are required for fully crystallized garnet, but this severely complicates fabrication due to alignment errors. However, Ce:TbIG had very good isolation $(11 \mathrm{~dB})$ without any seedlayer, and much higher ratios would be possible with only $7.35 \mathrm{~mm}$ lengths. Considering the convenience of onestep garnet deposition, Ce:TbIG (Figure $5 \mathrm{~d}$ ) is likely the best candidate for foundry-friendly integrated isolators on $500 \mathrm{~nm}$ SOI that can match the available integrated lasers of today.

\section{CONCLUSION}

Waveguide isolators on $500 \mathrm{~nm}$ SOI, matching the dimension and modal operation of currently available integrated lasers, can be realized with a high magnetic gyrotropy material like Ce:TbIG. Consistently high Faraday rotations of $-3200^{\circ} / \mathrm{cm}$ are achieved with the optimization of annealing temperature and tight control of bias voltage at the cerium target. The feasibility of $500 \mathrm{~nm}$ isolators has been demonstrated with prototype devices, and Ce:TbIG cladded isolators had the best trade-off between fabrication ease and isolation without an external field. The prototype device had an isolation ratio of 11 $\mathrm{dB}$, which can be improved simply by extending its length to $L_{\text {optimal }}$ at which a Stokes vector angle of $\pi$ is achieved between 
forward and backward propagating waves. The $1 \mathrm{D}$ waveguide isolators shown here can provide the high device density needed for large scale on-chip integration that is crucial for future silicon photonics.

\section{METHODS}

Ce:TbIG Growth and Characterization. Ce:TbIG thin films were sputter-deposited on double side polished silicon substrates $(10 \mathrm{~mm} \times 10 \mathrm{~mm})$ using reactive $\mathrm{RF}$ magnetron cosputtering at a base pressure of $2.5 \mathrm{mT}$ Torr in an ambient of $\mathrm{Ar}+\mathrm{O}_{2}$ mixture. The sputtering power of the cerium target was varied to change the concentration of cerium in the films. Post-deposition, the samples were subjected to ex situ rapid thermal annealing for $3 \mathrm{~min}$ in $10 \mathrm{sccm}$ of $\mathrm{O}_{2}$ at temperatures ranging from $700-1000{ }^{\circ} \mathrm{C}$. The thickness of the samples was $250 \pm 7 \mathrm{~nm}$, measured using a KLA Tencor P7 surface profilometer.

The X-ray spectra was acquired using a Bruker Discover D8 $2 \mathrm{D}$ diffractometer through a $\Theta-2 \Theta$ scan in the coupled mode. The X-ray data was further processed in JADE and the library file (PDF \#01-071-0697) for TbIG was used to identify the polycrystalline phases. The elemental compositional analysis was carried out using an Oxford EDS detector in a JEOL 6500 SEM under an electron beam with an accelerating voltage of $15 \mathrm{kV}$. The $\mathrm{M}-\mathrm{H}$ loops were measured in a Lakeshore Vibrating Sample Magnetometer $(-10 \mathrm{kOe}$ to +10 $\mathrm{kOe}$ ) at room temperature. The refractive index is measured using spectroscopic ellipsometry and the results are shown in Figure S3.

Device Fabrication. The devices were fabricated using ebeam lithography. Two layers of PMMA (positive resist) were spin-coated and patterned to make the lift-off mask on SOI for Ce:TbIG deposition. The bilayer PMMA was removed using a conventional lift-off procedure. A $100 \mathrm{~nm}$ thick $\mathrm{Si}_{x} \mathrm{~N}_{y}$ (having the same refractive index as garnet) was uniformly coated using ICP-CVD (Inductively Coupled Plasma Chemical Vapor Deposition) to eliminate the periodic loading effect. To pattern the Si waveguides, HSQ (hydrogen silesquioxane) was spun on the wafer and then etched using a STS-ICP dry etch tool down to the oxide. The scanning electron microscope images of the waveguides before and after rapid thermal annealing are shown in Figure S7. The crystallinity of Ce:TbIG on the silicon waveguides was verified using electron backscatter diffraction (EBSD), and the results are shown in Figure S8.

Optical Measurements. Faraday rotation measurements were carried out at $1550 \mathrm{~nm}$ infrared telecommunication $S$ - or C-bands with the laser source in the transmission setup and a unidirectional magnetic field. The direction of the field is alternated to measure the Faraday effect as a difference between the TE and TM modes. The acquired data from the photodetectors in $\mathrm{mV}$ is converted to degrees using a calibration step and normalized to the sample thickness to obtain the Faraday rotation in degrees $/ \mathrm{cm}$.

Device Measurements. The TE-TM mode conversion efficiency is measured by scanning the wavelength with a lockin amplifier on a cleaved sample to form a Fabry-Perot cavity. The direction of the saturating magnetic field is alternated during the measurement to effectively measure nonreciprocal effects. TE mode light is injected into the sample and the TM output is measured using a lock-in amplifier under three conditions: no magnetic field, after saturation in a magnetic field $(\sim 1.2 \mathrm{kOe})$ in the forward direction, and in the reverse direction. A polarimeter was used to observe and record the three Stokes parameters $\left(S_{1}, S_{2}\right.$, and $\left.S_{3}\right)$ and the azimuth.

\section{ASSOCIATED CONTENT}

\section{Supporting Information}

The Supporting Information is available free of charge on the ACS Publications website at DOI: 10.1021/acsphotonics.9b00707.

(1) Poincare sphere depicting the polarization states in NRMC (Figure S1); (2) Material characterization: XRD (Figure S2), refractive index, and extinction coefficient from ellipsometry (Figure S3), fraction $\mathrm{Fe}$ and substituted Ce concentration (Figure S4), and sputtering parameter comparison table (Table S1); (3) Device design and optical measurements: Beat-length simulation (Figure S5), polarimeter measurements on the device (Figure S6), and isolation ratio calculation from Stokes vector angle (Figure S7); (3) Crystallization analysis of Ce:TbIG on Si waveguides: SEM and EBSD of garnet on Si waveguides (Figures S8 and S9) (PDF)

\section{AUTHOR INFORMATION}

\section{Corresponding Authors}

*E-mail: srini162@umn.edu.

*E-mail: stadler@umn.edu.

ORCID ${ }^{\circ}$

Karthik Srinivasan: 0000-0002-1656-2169

David C. Hutchings: 0000-0002-3310-5971

Present Addresses

OHuaxun Ark Mobile Broadband Industrial Park, Chentian Industrial Zone, 1 Baotian Road, Xixiang, Ban'an District, Shenzhen, China 518000.

$\square 6901$ W 84th St., \#350, Bloomington, MN 55438, U.S.A.

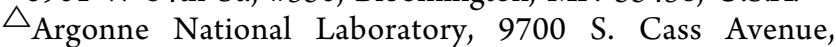
Lemont, IL 60439, U.S.A.

\section{Notes}

The authors declare no competing financial interest.

\section{ACKNOWLEDGMENTS}

This research was sponsored as a World Materials Network project by the U.S. National Science Foundation (DMR1210818) and the U.K. Engineering and Physical Sciences Research Council (EP/J018708/1) and also supported by the China Scholarship Council. Parts of this work were carried out in the Characterization Facility and the Minnesota Nano Center, University of Minnesota, which receives partial support from the NSF through the MRSEC and the National Nano Coordinated Infrastructure Network (NNCI Award Number 1542202) programs, respectively. The authors acknowledge the valuable support of waveguide patterning by the technical staff of the James Watt Nanofabrication Centre. Useful discussions with Dr. Barry Holmes are acknowledged by the authors.

\section{REFERENCES}

(1) Dai, D.; Bauters, J.; Bowers, J. E. Passive Technologies for Future Large-Scale Photonic Integrated Circuits on Silicon: Polarization Handling, Light Non-Reciprocity and Loss Reduction. Light: Sci. Appl. 2012, 1, e1.

(2) Thomson, D.; Zilkie, A.; Bowers, J. E.; Komljenovic, T.; Reed, G. T.; Vivien, L.; Marris-Morini, D.; Cassan, E.; Virot, L.; Fédéli, J.-M.; et al. Roadmap on Silicon Photonics. J. Opt. 2016, 18, 073003. 
(3) Jalas, D.; Petrov, A.; Eich, M.; Freude, W.; Fan, S.; Yu, Z.; Baets, R.; Popović, M.; Melloni, A.; Joannopoulos, J. D.; et al. What Is and What Is Not - an Optical Isolator. Nat. Photonics 2013, 7, 579582.

(4) Huang, D.; Pintus, P.; Bowers, J. E. Towards Heterogeneous Integration of Optical Isolators and Circulators with Lasers on Silicon [Invited]. Opt. Mater. Express 2018, 8, 2471.

(5) Lamponi, M.; Keyvaninia, S.; Jany, C.; Poingt, F.; Lelarge, F.; De Valicourt, G.; Roelkens, G.; Van Thourhout, D.; Messaoudene, S.; Fedeli, J. M.; et al. Low-Threshold Heterogeneously Integrated InP/ SOI Lasers with a Double Adiabatic Taper Coupler. IEEE Photonics Technol. Lett. 2012, 24, 76-78.

(6) Bi, L.; Hu, J.; Jiang, P.; Kim, D. H.; Dionne, G. F.; Kimerling, L. C.; Ross, C. A. On-Chip Optical Isolation in Monolithically Integrated Non-Reciprocal Optical Resonators. Nat. Photonics 2011, $5,758-762$.

(7) Ghosh, S.; Keyvaninia, S.; Shirato, Y.; Mizumoto, T.; Roelkens, G.; Baets, R. Optical Isolator for TE Polarized Light Realized by Adhesive Bonding of Ce:YIG on Silicon-on-Insulator Waveguide Circuits. IEEE Photonics J. 2013, 5, 6601108.

(8) Shoji, Y.; Miura, K.; Mizumoto, T. Optical Nonreciprocal Devices Based on Magneto-Optical Phase Shift in Silicon Photonics. J. Opt. 2016, 18, 013001.

(9) Zhang, C.; Dulal, P.; Stadler, B. J. H.; Hutchings, D. C. Monolithically-Integrated TE-Mode 1D Silicon-on-Insulator Isolators Using Seedlayer-Free Garnet. Sci. Rep. 2017, 7, 1-8.

(10) Stadler, B. J. H.; Mizumoto, T. Integrated Magneto-Optical Materials and Isolators: A Review. IEEE Photonics J. 2014, 6, 1-15.

(11) Stadler, B. J. H.; Hutchings, D. C. Sputter-Deposited MagnetoOptical Garnet for All-Mode (Transverse Electric/Transverse Magnetic) Faraday Rotators. MRS Bull. 2018, 43, 430-435.

(12) Huang, D.; Pintus, P.; Zhang, C.; Shoji, Y.; Mizumoto, T.; Bowers, J. E. Electrically Driven and Thermally Tunable Integrated Optical Isolators for Silicon Photonics. IEEE J. Sel. Top. Quantum Electron. 2016, 22, 271-278.

(13) Karki, D.; Stenger, V.; Pollick, A.; Levy, M. Thin-Film Magnetless Faraday Rotators for Compact Heterogeneous Integrated Optical Isolators. J. Appl. Phys. 2017, 121, 233101.

(14) Srinivasan, K.; Stadler, B. J. H. Magneto-Optical Materials and Designs for Integrated TE- and TM-Mode Planar Waveguide Isolators: A Review [Invited]. Opt. Mater. Express 2018, 8, 3307.

(15) Ghosh, S.; Keyvaninia, S.; Shoji, Y.; Van Roy, W.; Mizumoto, T.; Roelkens, G.; Baets, R. G. Compact Mach-Zehnder Interferometer Ce:YIG/SOI Optical Isolators. IEEE Photonics Technol. Lett. 2012, 24, $1653-1656$

(16) Pintus, P.; Huang, D.; Zhang, C.; Shoji, Y.; Mizumoto, T.; Bowers, J. E. Microring-Based Optical Isolator and Circulator with Integrated Electromagnet for Silicon Photonics. J. Lightwave Technol. 2017, 35, 1429-1437.

(17) Takei, R.; Mizumoto, T. Design and Simulation of Silicon Waveguide Optical Circulator Employing Nonreciprocal Phase Shift. Jpn. J. Appl. Phys. 2010, 49, 052203.

(18) Huang, D.; Pintus, P.; Bowers, J. E. Towards Heterogeneous Integration of Optical Isolators and Circulators with Lasers on Silicon [Invited]. Opt. Mater. Express 2018, 8, 2471.

(19) Piggott, A. Y.; Lu, J.; Lagoudakis, K. G.; Petykiewicz, J.; Babinec, T. M.; Vučković, J. Inverse Design and Demonstration of a Compact and Broadband On-Chip Wavelength Demultiplexer. Nat. Photonics 2015, 9, 374-377.

(20) Dai, D.; Wang, J.; Shi, Y. Silicon Mode (de)Multiplexer Enabling High Capacity Photonic Networks-on-Chip with a SingleWavelength-Carrier Light. Opt. Lett. 2013, 38, 1422.

(21) Xia, F.; Sekaric, L.; Vlasov, Y. Ultracompact Optical Buffers on a Silicon Chip. Nat. Photonics 2007, 1, 65-71.

(22) Keyvaninia, S.; Roelkens, G.; Van Thourhout, D.; Jany, C.; Lamponi, M.; Le Liepvre, A.; Lelarge, F.; Make, D.; Duan, G.-H.; Bordel, D.; et al. Demonstration of a Heterogeneously Integrated IIIV/SOI Single Wavelength Tunable Laser. Opt. Express 2013, 21, 3784-3792.
(23) Fratello, V.J.; Licht, S.J.; Brandle, C.D. Innovative Improvements in Bismuth-Doped Rare-Earth Iron Garnet Faraday Rotators. IEEE Trans. Magn. 1996, 32, 4102-4107.

(24) Shirasaki, M.; Asama, K. Compact Optical Isolator for Fibers Using Birefringent Wedges. Appl. Opt. 1982, 21, 4296-4299.

(25) Zhang, Y.; Du, Q.; Wang, C.; Fakhrul, T.; Liu, S.; Deng, L.; Huang, D.; Pintus, P.; Bowers, J.; Ross, C. A.; et al. Monolithic Integration of Broadband Optical Isolators for Polarization-Diverse Silicon Photonics. Optica 2019, 6, 473.

(26) Dötsch, H.; Bahlmann, N.; Zhuromskyy, O.; Hammer, M.; Wilkens, L.; Gerhardt, R.; Hertel, P.; Popkov, A. F. Applications of Magneto-Optical Waveguides in Integrated Optics: Review. J. Opt. Soc. Am. B 2005, 22, 240.

(27) Ando, K.; Okoshi, T.; Koshizuka, N. Waveguide Magneto optic Isolator Fabricated by Laser Annealing. Appl. Phys. Lett. 1988, 53, 46.

(28) Hutchings, D. C.; Holmes, B. M.; Zhang, C.; Dulal, P.; Block, A. D.; Sung, S. Y.; Seaton, N. C. A.; Stadler, B. J. H. Quasi-PhaseMatched Faraday Rotation in Semiconductor Waveguides with a Magnetooptic Cladding for Monolithically Integrated Optical Isolators. IEEE Photonics J. 2013, 5 (6), 6602512.

(29) Dulal, P.; Block, A. D.; Gage, T. E.; Haldren, H. A.; Sung, S. Y.; Hutchings, D. C.; Stadler, B. J. H. Optimized Magneto-Optical Isolator Designs Inspired by Seedlayer-Free Terbium Iron Garnets with Opposite Chirality. ACS Photonics 2016, 3, 1818-1825.

(30) Dulal, P.; Gage, T. E.; Block, A. D.; Cofell, E.; Hutchings, D. C.; Stadler, B. J. H. Sputter-Deposited Seedlayer-Free Cerium-Doped Terbium Iron Garnets for SOI Waveguide Isolators. 2016 IEEE Photonics Conference (IPC); IEEE, 2016; pp 773-774.

(31) Srinivasan, K.; Gage, T. E.; Stadler, B. J. H. Seed-Layer Free Cerium-Doped Terbium Iron Garnet on Non-Garnet Substrates for Photonic Isolators. Conference on Lasers and Electro-Optics; OSA Technical Digest; Optical Society of America: San Jose, CA, 2018; p SW4I.5.

(32) Sung, S. Y.; Sharma, A.; Block, A.; Keuhn, K.; Stadler, B. J. H. Magneto-Optical Garnet Waveguides on Semiconductor Platforms: Magnetics, Mechanics, and Photonics. J. Appl. Phys. 2011, 109, 20092012.

(33) Block, A. D.; Dulal, P.; Stadler, B. J. H.; Seaton, N. C. A. Growth Parameters of Fully Crystallized YIG, Bi:YIG, and Ce:YIG Films With High Faraday Rotations. IEEE Photonics J. 2014, 6, 1-8.

(34) Sun, X. Y.; Du, Q.; Goto, T.; Onbasli, M. C.; Kim, D. H.; Aimon, N. M.; Hu, J.; Ross, C. A. Single-Step Deposition of CeriumSubstituted Yttrium Iron Garnet for Monolithic On-Chip Optical Isolation. ACS Photonics 2015, 2, 856-863.

(35) Gage, T. E.; Dulal, P.; Solheid, P. A.; Flannigan, D. J.; Stadler, B. J. H. Si-Integrated Ultrathin Films of Phase-Pure Y3Fe5O12 (YIG) via Novel Two-Step Rapid Thermal Anneal. Mater. Res. Lett. 2017, 5, $379-385$.

(36) Gomi, M.; Furuyama, H.; Abe, M. Strong Magneto optical Enhancement in Highly Ce substituted Iron Garnet Films Prepared by Sputtering. J. Appl. Phys. 1991, 70, 7065-7067.

(37) Winkler, G. Magnetic Garnets; Vieweg, 1981.

(38) Phillips, C. R.; Langrock, C.; Chang, D.; Lin, Y. W.; Gallmann, L.; Fejer, M. M. Apodization of Chirped Quasi-Phasematching Devices. J. Opt. Soc. Am. B 2013, 30, 1551. 